\title{
Ko143 Reverses MDR in Glioblastoma via Deactivating P-Glycoprotein, Sensitizing a Resistant Phenotype to TMZ Treatment
}

\author{
SAMUEL D. LUSTIG ${ }^{1 *}$, SRAVAN K. KODALI ${ }^{2 *}$, SHARON L. LONGO ${ }^{3}$, \\ SOMANATH KUNDU ${ }^{4}$ and MARIANO S. VIAPIANO ${ }^{3}$ \\ ${ }^{1}$ University School of Nashville, Nashville, TN, U.S.A.; \\ ${ }^{2}$ Christian Brothers Academy, Syracuse, NY, U.S.A.; \\ ${ }^{3}$ Departments of Neurosurgery and Neuroscience and Physiology, \\ SUNY Upstate Medical University, Syracuse, NY, U.S.A.; \\ ${ }^{4}$ Department of Neuroscience \& Physiology, SUNY Upstate Medical University, Syracuse, NY, U.S.A.
}

\begin{abstract}
Background/Aim: Over-expression of both $P$-glycoprotein (P-gp) and Breast Cancer Resistance Protein $(B C R P)$ has been associated with multidrug-resistance in glioblastoma (GBM). Though previously studied broadspectrum inhibitors of drug efflux pumps have failed to progress in clinical studies due to in vivo toxicity, research into clinically viable targeted inhibitors is needed. This study evaluated the effects of Kol43, a non-toxic analog of fumitremorgin $C$, on temozolomide (TMZ) efficacy in resistant glioblastoma stem cells. Materials and Methods: We used ATP-Glo assay to determine cell viabilities and flow cytometry to perform cell cycle analysis. Comparative gene expression was analysed through RT-qPCR. Results: TMZ $I C_{50}$ decreased $41.07 \%(p<0.01)$ in the resistant phenotype when delivered in combination with Ko143. Additionally, the TMZ-resistant phenotype (GBM146) displayed 44-fold greater P-gp expression than the TMZ-sensitive phenotype (GBM9) $(p<0.01)$, yet a 0.6-fold lower BCRP expression. Kol43 potentiates TMZ efficacy and likely inhibits $P$ glycoprotein more potently than previously indicated. Conclusion: Further development of non-toxic, targeted inhibitors of drug efflux pumps for use in combinatorial chemotherapy may improve glioblastoma patient prognosis.
\end{abstract}

This article is freely accessible online.

*These Authors contributed equally to this study.

Correspondence to: Sravan K. Kodali, 340 Rosewood Cir., Canastota, NY 13032, U.S.A. Tel: +1 7036891649, e-mail: sravan.kodali@cbasyr.org

Key Words: Ko143, combinatorial treatment, P-glycoprotein, BCRP, drug resistance, temozolomide.
Glioblastoma (GBM) is the most common primary malignant brain tumor in adults. Adult GBM patients face an average prognosis of only 15 months (1). Most commonly, treatment is initiated with intracranial surgery to achieve maximal tumor resection (2). However, surgery remains extremely difficult and is generally unable to eliminate the cancer completely. Surgical resection is followed by chemotherapy and radiation treatment to the site of cancerous growth. This multimodal therapy regimen provides low efficacy in extending patient survival, highlighted by a 4-5\% 5-year survival rate for GBM patients (3). Temozolomide (TMZ) has been the standard of care chemotherapeutic for GBM patients since 2005 (4). Unlike many other chemotherapeutic drugs, TMZ possesses the ability to penetrate the blood-brain barrier (BBB) (5). As an alkylating agent, TMZ adds a methyl group to $\mathrm{O}^{6}$-guanine, $\mathrm{N}^{7}$-guanine, and $\mathrm{N}^{3}$-adenine bases, inducing DNA lesions (6). These lesions are cytotoxic due to their role in obstructing DNA replication (7). During DNA replication, DNA polymerase mispairs the methyl adducts, signaling a repetitive cycle of unsuccessful DNA mismatch repair (MMR) (8). Ineffective MMR contributes to the occurrence of single- and double-strand breaks within the DNA, triggering cell cycle arrest and apoptosis (9). Despite the widespread use of TMZ, over $50 \%$ of GBM patients are unresponsive to the drug due to various resistance mechanisms (10). Cancerous stem cells (CSCs) are equipped with many tumorigenic mechanisms, including the ability to self-renew and differentiate into fast-dividing cancer cell progeny (11). CSCs make up a minor percentage of the cellular composition of GBM tumors but are the primary culprit of cancer invasion and recurrence (12). Multidrug resistance (MDR) is often one of the characteristics of CSCs (13). CSCs are able to evade the effects of chemotherapeutics via a multitude of MDR 
<smiles>COc1ccc2c3c([nH]c2c1)[C@@H](C=C(C)C)N1C(=O)[C@@H]2CCCN2C(=O)[C@H]1C3</smiles>

Fumitremorgin C<smiles>COc1ccc2c3c([nH]c2c1)C(CC(C)C)N1C(=O)[C@H](CCC(=O)OC(C)(C)C)NC(=O)C1C3</smiles>

Ko143

Figure 1. Ko143 is a non-toxic analog of fumitremorgin C. Ko143 differs from fumitremorgin $C$ in three positions. It does not contain the E-ring acquired from amino acid, L-proline, and features $t$-Bu-protected L-glutamic amino acid side chains as a C-6 substituent. Ko143 also has an isobutyl substituent at C-3, which is the C-21, C-22 saturated equivalent of the natural side chain found in fumitremorgin $C$ (25).

mechanisms. For example, CSCs have the ability to activate signaling pathways that retain an undifferentiated state with high resistance to chemotherapy (14). Additionally, GBM stem cells (GSCs) can repair DNA lesions caused by TMZ through the use of $\mathrm{O}^{6}$-methylguanine methyltransferase (MGMT), which is able to remove methyl adducts (15).

ATP-binding cassette $(\mathrm{ABC})$ transporters contribute to a fundamental mechanism of MDR within GSCs that lowers the efficacy of TMZ. These ABC transporters, also known as drug efflux pumps, reduce drug-induced cytotoxicity by decreasing the intracellular content of the drug (16). In this study, two specific ABC transporters were studied within GSCs: Pglycoprotein (P-gp/ABCB1/MDR1), a $170 \mathrm{kDA}$ transmembrane glycoprotein; and breast cancer resistance protein (BCRP/ABCG2/CDw338), a 70kDA monomer and a $140 \mathrm{kDA}$ dimer, formed by disulfide bonds (17). While ABCB1 contains two nucleotide-binding domains (NBDs) and two transmembrane domains (TMDs), allowing for full functionality as a monomeric protein, $\mathrm{ABCG} 2$ requires dimerization, since it only encompasses one NBD and one TMD (18). In both, a substrate binds to the substrate-binding domains (SBDs), and dimerization of the two nucleotide-binding domains (NBDs) occurs via ATP hydrolysis (19). This process provides energy for both proteins to transition between conformational states, which is essential to the transport cycle. Various substrates may compete for these sites, including TMZ. Previous research has outlined the effects of P-gp and BCRP in GBM. Importantly, TMZ was shown to be a substrate of both proteins, leading to the conclusion that TMZ is actively effluxed out of the cell by both ABCB1 and ABCG2 (20).

Multiple dual inhibitors of both P-gp and BCRP have been tested clinically, yet none have achieved success in reversing multidrug resistance safely for patients. Tariquidar, a dual inhibitor of P-gp and BCRP and a widely studied drug in the laboratory, proved to have minimal antitumor effects in patients with lung, ovarian, and cervical cancers when paired with docetaxel (21). Another well-known dual inhibitor, elacridar, was tested in phase I clinical trials but led to a common toxicity of neutropenia, a low count of neutrophils that significantly increases an individual's susceptibility to life-threatening infections. Additionally, increased systemic exposure to the co-administered chemotherapeutic, docetaxel, led to no further clinical development (22). Disappointing clinical trials for both of these drugs evoked the search for a less toxic and more clinically promising inhibitor. One heavily researched and selective BCRP inhibitor, fumitremorgin C (FTC), displays high neurotoxicity and has been proven to have minimal effects on P-gp (23). Although the inhibition of MDR through FTC would not be viable for in vivo treatment, a nontoxic analog, Ko143, was considered a top candidate due to its inhibitory activity against BCRP and P-gp at high concentrations $(>1 \mu \mathrm{M})(24)$. The molecular structures of FTC and Ko143 are compared in Figure 1. Ko143 ultimately stood out as the least researched dual inhibitor of P-gp and BCRP, prompting its selection in this study.

Since Ko143's mechanism of action has been shown to reverse MDR, the compound was tested as a potential TMZsensitizing agent. This study provides a detailed assessment of TMZ+Ko143 treatment on resistant glioblastoma phenotypes and analyzes the role P-gp and BCRP play in promoting MDR in the presence and absence of Ko143.

\section{Materials and Methods}

Reagents. Ko143 hydrate (K2144) and temozolomide (T2577) were purchased from Sigma-Aldrich (St. Louis, MO, USA). The MTS reagent used to measure cellular metabolism (CellTiter Cell Proliferation Assay) and the ATP-Glo reagent that measures the levels of ATP in live cells (CellTiterGlo Viability Assay) were purchased as kits (Promega Corp., Madison, WI, USA). For RTqPCR, forward and reverse primer oligonucleotides for $A B C B 1$ and $A B C G 2$, as well as for $G A P D H$, a housekeeping gene used as control, were purchased from Integrated DNA technologies, Inc., 
(Coralville, IA, USA). Skeletal structures of Ko143 and fumitremorgin $\mathrm{C}$ were generated with King Draw Chemical Structure Editor (King Draw, Corp., Qingdao, Shandong, PR China).

Cell culture. For all assays except for flow cytometry, 5,000 cells were plated per well and suspended in $50 \mu \mathrm{l}$ of cell culture medium. A total of 10,000 cells were plated per well for flow cytometry. The GSC medium, specifically designed by SUNY Upstate Medical University for patient-derived GSC culturing, was composed of the following: DMEM/F12 50/50 (500 ml) supplemented with Lglutamine $100 \times(5 \mathrm{ml})$, B27 supplement $50 \times(10 \mathrm{ml})$, epidermal growth factor $(20 \mathrm{ng} / \mathrm{ml}$ final concentration), basic fibroblast growth factor $(20 \mathrm{ng} / \mathrm{ml}$ final concentration), porcine heparin $(50 \mathrm{ng} / \mathrm{ml}$ final concentration), and penicillin/streptomycin $50 \times(5 \mathrm{ml})$. GBM9 cell cultures were genetically engineered via lentiviral transduction to express enhanced green fluorescent protein (eGFP). This provided the study with a unique way to image the control cell line using fluorescence microscopy (Figure 2).

MTS assay. The initial screening experiment was performed using an MTS reduction assay, which tested multiple variations of the combination therapy. Weidner et al. showed that Ko143 concentrations for P-gp and BCRP inhibition should be $\geq 1 \mu \mathrm{M}$. Thus, in the MTS experiment, Ko143 was applied at concentrations of 1.5 $\mu \mathrm{M}$ and $5 \mu \mathrm{M}$ to compare the effects of each concentration in various samples. Certain samples were treated with the combination of Ko143 and TMZ, whose concentration ranged from $50 \mu \mathrm{M}$ to $400 \mu \mathrm{M}$. These concentrations were initially chosen from a review of relevant literature. Controls were composed of cells treated with TMZ alone, Ko143 alone, and no drug. A total of 5,000 cells were plated in each well, and the duration of the experiment was $48 \mathrm{~h}$. MTS solution was added at $20 \mu \mathrm{l}$ per $100 \mu \mathrm{l}$ volume in each well. Four hours later, absorbance measurements of the resulting formazan dye (bioreduced MTS), were taken using an Infinity 200 Pro multiwell plate reader from Tecan Trading AG (Zürich, Switzerland). These measurements are representative of cell viability since NADPH-dependent dehydrogenase enzymes are required to reduce MTS, and thus only metabolically active cells will have high absorbance. After adjusting for background noise, absorbance values were averaged between triplicate samples and computed relative to controls. Although data were derived from this experiment, it was later discovered that a major technical artifact misrepresented the data. The results are not shown for this reason, but a detailed description of this complication can be found in the discussion.

ATP-Glo assay. As a flash-type luminescence assay based on the detection of ATP in live cells, ATP-Glo provides more sensitivity and more accuracy than the MTS reduction assay. The assay was performed to determine the half-maximal inhibitory concentration $\left(\mathrm{IC}_{50}\right)$ values of TMZ for different doses of $\mathrm{TMZ}+\mathrm{Ko} 143$ combination treatment and for TMZ alone treatment. The effects of Ko143 alone on cell viability were also assessed to determine the possible cytotoxicity of the drug. Two GBM cell lines (GBM9 and GBM146) were left untreated (resuspended in GSC culture medium alone) or treated with Ko143 $(5 \mu \mathrm{M})$, TMZ $(100 \mu \mathrm{M}-800 \mu \mathrm{M})$, or Ko143 $(5 \mu \mathrm{M})+\mathrm{TMZ}(100 \mu \mathrm{M}-800 \mu \mathrm{M})$. Three wells were used for each treatment for both GBM9 and GBM146 cells $(5,000$ cells/well), and the duration of the experiment was $72 \mathrm{~h}$. Luciferin oxidation, through the addition of ATP, $\mathrm{Mg}^{2+}$, and luciferase, was used to produce oxyluciferin, providing quantifiable luminescence.
Luminescence signals were measured using a Synergy-2 multiwell plate reader purchased from Agilent Technologies (Santa Clara, CA, USA). Luminescence values were corrected for background noise, and normalized as percentages relative to controls. Since luciferin oxidation requires ATP, which is an indicator of metabolically active cells, the detection of luminescence correlates to cell viability. One line was chosen as the negative control as it proved to be highly sensitive to TMZ (GBM9), whereas the other cell line was chosen as the experimental group due to its high TMZ resistance (GBM146).

Cell cycle analysis. Cell cycle analysis, through flow cytometry, was conducted to confirm the non-toxic effects of Ko143. An increase in the number of cells in sub- $\mathrm{G}_{0} / \mathrm{G}_{1}$ phase in treated samples compared to control samples was representative of an increase in apoptosis/necrosis. GBM146 cells treated with Ko143 $(5 \mu \mathrm{M})$ were analyzed alongside controls via flow cytometry. Cells were fixed with $70 \%$ ethanol, and $48 \mathrm{~h}$ after cell plating their DNA was stained with propidium iodide. The samples were gated to measure only the DNA content of cells exhibiting an appropriate size; debris and cells displaying an abnormal size were discarded.

Reverse transcription quantitative real-time $P C R$. The relative expression of the $A B C B 1$ and $A B C G 2$ genes was quantified in each cell line to analyze possible mechanisms that contribute to Ko143's potentiation of the antineoplastic effects of TMZ. The $A B C B 1, A B C G 2$, and $G A P D H$ genes were relatively quantified in GBM9 and GBM146 cells (GAPDH was analyzed as a housekeeping control gene) using gene-specific primers (Table I). Once other PCR reagents were added, samples were processed using a CFX96 Touch Deep Well Real-Time qPCR Detection System (Bio-Rad Laboratories Inc., Hercules, CA, USA). Data were analyzed using the instrument's software, and further analysis was performed using Microsoft Excel (Microsoft Corp., Redmond, WA, USA) and GraphPad Prism (GraphPad Software, Inc., San Diego, CA, USA). Relative quantifications of the genes were computed using the following equation:

$\mathrm{RQ}=2^{-\Delta \Delta \mathrm{C}_{\mathrm{t}}}$

where $\mathrm{C}_{\mathrm{t}}=$ threshold for detection of a desired gene, $\Delta \mathrm{C}_{\mathrm{t}}=$ difference in expression between the desired gene and a housekeeping gene $(G A P D H)$, and $\Delta\left(\Delta C_{t}\right)=$ difference in expression for the desired gene (minus the housekeeping gene) between control and treated conditions.

Imaging. An Olympus IX-71 Inverted Fluorescence Microscope (Olympus Corp., Tokyo, Japan) equipped with a QImaging MicroPublisher CCD Microscope Camera (Teledyne Quantitative Imaging Corporation, Surrey, BC, Canada) and the CoolLED pE300 (CoolLED Ltd., Andover, UK) illumination system was used to image tumor cells. Images of GBM146 and GBM9 cells were captured under brightfield illumination at $20 \times$ magnification. Additionally, GBM9 cultures, which were genetically engineered to express eGFP, were imaged under fluorescence illumination at 20x magnification.

Statistical analysis. GraphPad Prism version 9.0.1 was used to graph and analyze data and to complete statistical tests. Raw data were computed on Microsoft Excel 2021 and later transferred to GraphPad Prism for further testing. Calculla 2.0 (Kemu Studios Ltd., West Yorkshire, UK) was utilized for nonlinear regression 
Representative images of glioblastoma stem cells (GBM9 cell line)

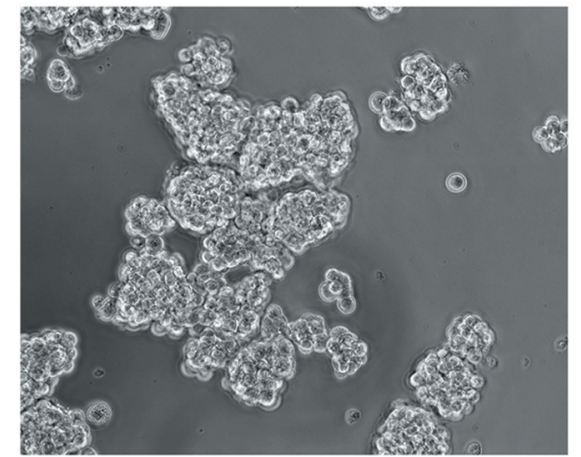

High cell density
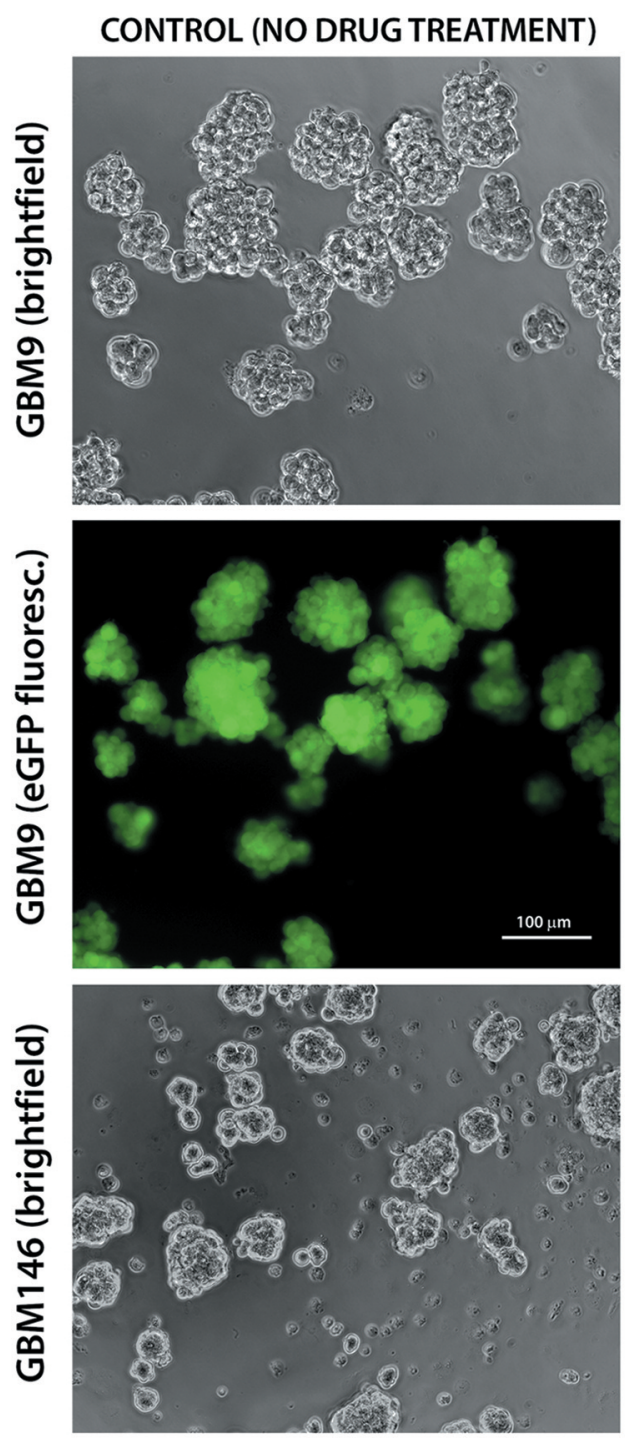

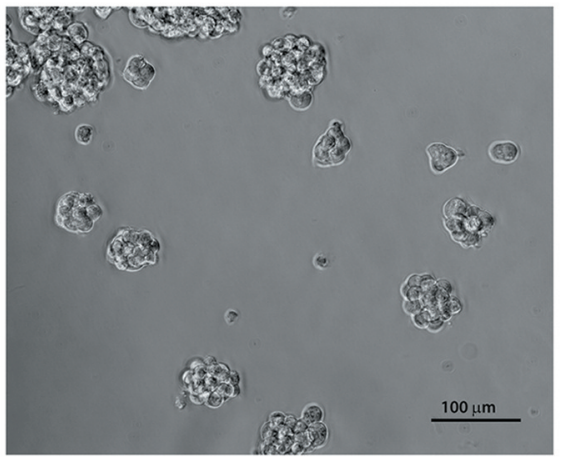

Low cell density

TEMOZOLOMIDE $400 \mu \mathrm{M}$
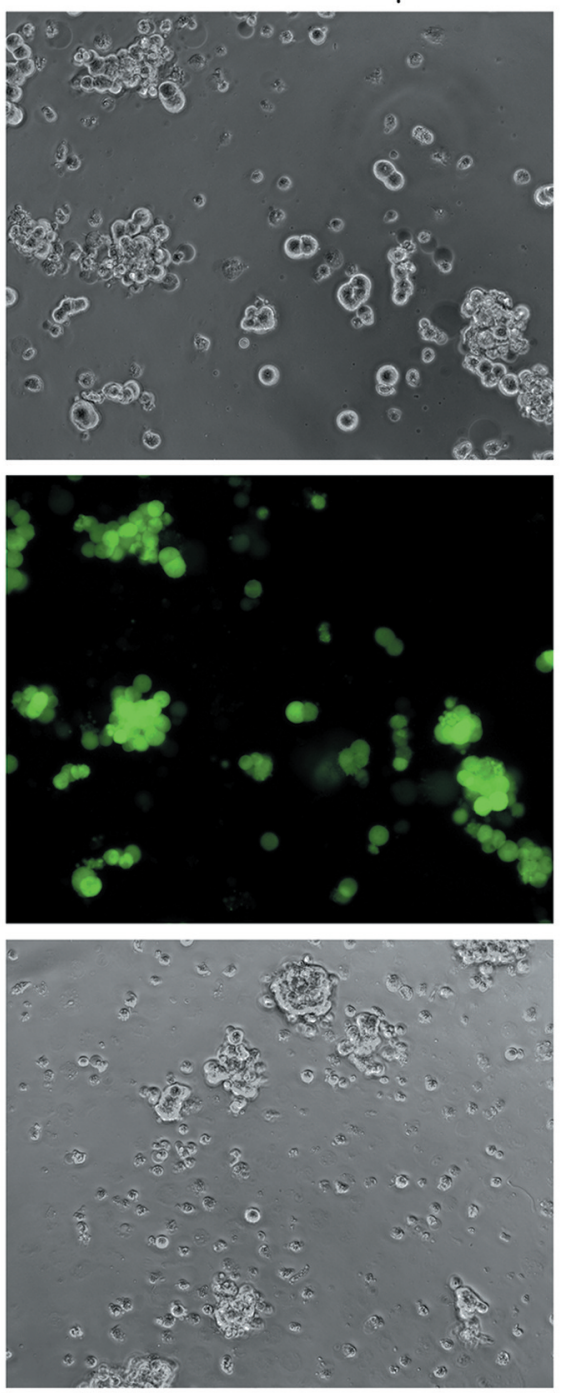

Figure 2. Temozolomide (TMZ)-induced apoptosis in chemosensitive and chemoresistant glioblastoma stem cells. Cell cultures from ATP-Glo assay were imaged with the Olympus IX71 inverted microscope at 20x magnification 72 h after treatment. GBM9 was engineered via lentiviral transduction to express the enhanced green fluorescence protein (eGFP). The top two panels use brightfield microscopy to visualize regions of GBM9 neurospheres characterized by low density or high density. Samples were plated at 5,000 cells/well. The other panels compare the cell killing effects of TMZ in the TMZ-sensitive (GBM9) and TMZ-resistant (GBM146) phenotypes through both brightfield (GBM9 and GBM146) and fluorescence microscopy (GBM9). 
Table I. Gene-specific oligonucleotide primer sequences.

\begin{tabular}{|c|c|}
\hline Gene & Reverse and forward oligonucleotide sequences \\
\hline$A B C B 1$ & $\begin{array}{l}\text { Forward primer=TGCTGGTTGCTGCTTAC; } \\
\text { Reverse primer }=\text { GCCTATCTCCTGTCGCATTATAG }\end{array}$ \\
\hline$A B C G 2$ & $\begin{array}{l}\text { Forward primer=ACGAACGGATTAACAGGGTCA; } \\
\text { Reverse primer=CTCCAGACACACCACGGAT }\end{array}$ \\
\hline GAPDH & $\begin{array}{l}\text { Forward primer=TTGCCCTCAACGACCACTTT; } \\
\text { Reverse primer=TGGTCCAGGGGTCTTACTCC }\end{array}$ \\
\hline
\end{tabular}

analysis. All graphs exhibited determination coefficients $>0.99$. $\mathrm{IC}_{50}$ values were computed with both GraphPad Prism and Desmos (Desmos Inc., San Diego, CA, USA). All graphs other than those used to analyze flow cytometry data were created using these two programs. Flow cytometry data were directly computed and graphed with a Millipore Guava Muse (Luminex Corp., Austin, TX, USA). Data were considered statistically significant only if the $p$ value was $<0.05$ for each statistical comparison.

\section{Results}

Kol43 sensitizes TMZ-resistant glioblastoma phenotype dosedependently. TMZ-mediated cytotoxicity increased corresponding to increases in dose concentrations and a $>50 \%$ decrease in the viability of both GBM9 and GBM146 cells was observed. The percent cell viability after treatment with $\mathrm{TMZ}$ or $\mathrm{TMZ}+\mathrm{Ko} 143$ was used to generate graphs with nonlinear regression. Three graphs were created to represent each of the three replicate rows of wells for both the TMZ alone treatment and the TMZ+Ko143 treatment. This resulted in the development of six graphs (each with an $r^{2}>0.99$ ) from which we computed $\mathrm{TMZ} \mathrm{IC}_{50}$ values and analyzed them in GraphPad Prism with an unpaired two-tailed t-test, producing $p$-values for the comparison of $\mathrm{IC}_{50} \mathrm{~s}$ of each treatment for both GBM9 and GBM146 cells. The $\mathrm{IC}_{50}$ value in GBM146 cells when TMZ was used in combination with $5 \mu \mathrm{M}$ Ko143 was $470.7 \mu \mathrm{M}$. The $\mathrm{IC}_{50}$ value when $\mathrm{TMZ}$ was used alone was $798.8 \mu \mathrm{M}$, which was much higher than that when TMZ was combined with $\mathrm{Ko143}$. The $\mathrm{IC}_{50}$ values for $\mathrm{TMZ}$ alone and in combination with Ko143 in GBM9 cells were also analysed. The combination treatment resulted in an $\mathrm{IC}_{50}$ value of $141.1 \mu \mathrm{M}$, and the $\mathrm{IC}_{50}$ value for $\mathrm{TMZ}$ alone was calculated to be $148.5 \mu \mathrm{M}$. These $\mathrm{IC}_{50}$ values were also analyzed by t-tests to determine statistical significance. The variation in $\mathrm{IC}_{50}$ values in GBM9 cells treated with $\mathrm{TMZ}$ alone $v s$. TMZ+Ko143 $(5 \mu \mathrm{M})$ was statistically nonsignificant, with $p=0.7869$. However, in GBM146 cells, an identical analysis showed that the difference in the TMZ $\mathrm{IC}_{50}$ values is statistically significant, with $p=0.0015$. Comparison of $\mathrm{TMZ} \mathrm{IC}_{50}$ values tested on GBM9 and GBM146 cells indicated a 5.37-fold greater TMZ sensitivity in GBM9

\section{GBM9 cell viability: TMZ vs. TMZ+Ko143 - after 72h}

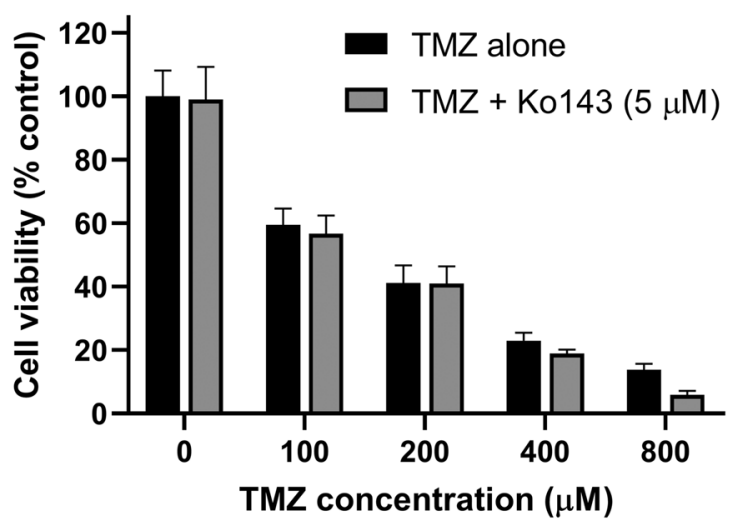

GBM146 cell viability: TMZ vs. TMZ+Ko143 - after 72h

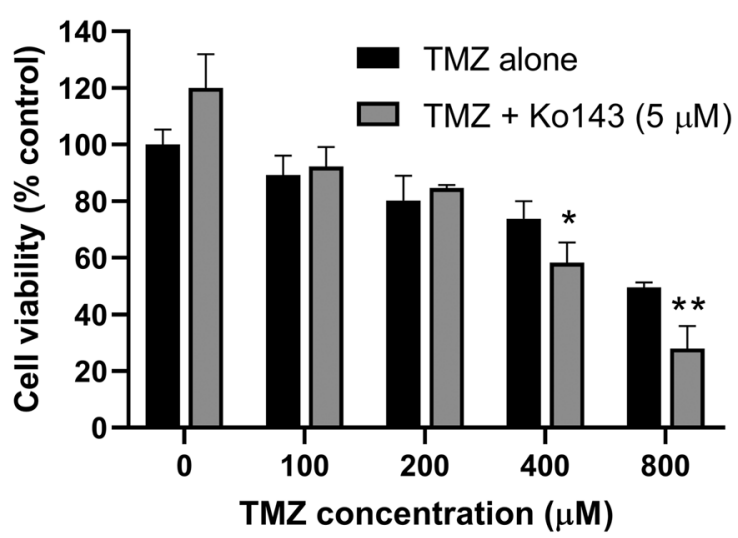

Figure 3. Ko143 treatment sensitizes GBM146 to temozolomide (TMZ) dose-dependently. Cell viability of both GBM phenotypes was determined with the ATP-Glo assay. GBM9 and GBM146 cells were left untreated (resuspended in GSC culture medium alone) or treated with Ko143 (5 $\mu M)$, TMZ $(100 \mu M-800 \mu M)$, or Kol43 $(5 \mu M)+T M Z(100 \mu M-800 \mu M)$. Triplicates were used for each treatment type and consisted of 5,000 cells/well. The duration of the experiment was $72 \mathrm{~h}$. Luminescence signals were measured using the Synergy-2 multiwell plate reader. Cell viability changes between the TMZ and TMZ+Ko143 treatment were statistically significant at TMZ doses of $400 \mu M$ and $800 \mu M$ in GBM146. Significance was recognized by t-test at $* p<0.05$ and $* * p<0.01$.

compared to GBM146 ( $p<0.0001)$. The $41 \%$ lower $\mathrm{IC}_{50}$ value of TMZ used in combination with Ko143 (5 $\mu \mathrm{M})$ relative to the $\mathrm{IC}_{50}$ of $\mathrm{TMZ}$ alone supports our hypothesis that Ko143 improves the efficacy of TMZ in chemoresistant GSCs (represented by GBM146 cells) but not to a similar extent in highly chemosensitive GSCs (represented by GBM9 cells) if at all (Table II). In Figure 3, TMZ alone and the TMZ+Ko143 (5 $\mu \mathrm{M}$ ) combination treatment are analyzed for their differences in GBM9 and GBM146. 
Table II. Regression analysis of temozolomide (TMZ) $I C_{50}$ in TMZResistant (R) and TMZ-Sensitive (S) GSCs.

\begin{tabular}{lcc}
\hline $\begin{array}{l}\text { Cell type } v s . \\
\text { TMZ IC }_{50}\end{array}$ & $\begin{array}{c}\text { TMZ alone } \\
\text { treatment }(\mu \mathrm{M})\end{array}$ & $\begin{array}{c}\text { TMZ+Ko143 } \\
\text { treatment }(\mu \mathrm{M})\end{array}$ \\
\hline GBM146 cells (TMZ-R) & 798.8 & 470.7 \\
GBM9 cells (TMZ-S) & 148.5 & 141.1 \\
\hline
\end{tabular}

Percentage of cells in sub- $G_{0} / G_{1}$ phase unaffected by Kol43 alone in TMZ-resistant glioblastoma phenotype. The ATPGlo assay showed that the $\mathrm{IC}_{50}$ of $\mathrm{TMZ}$ combined with Ko143 was lower than that of TMZ alone in GBM146 cells, directing us to test the effects of TMZ alone $(400 \mu \mathrm{M})$, Ko143 alone $(5 \mu \mathrm{M})$, and Ko143 $(5 \mu \mathrm{M})+\mathrm{TMZ}(400 \mu \mathrm{M})$ on GBM146 cells via flow cytometry. The cell killing ability of TMZ was once more confirmed through this assay, as $34 \%$ of cells treated with TMZ were in sub- $\mathrm{G}_{0} / \mathrm{G}_{1}$ phase (recognized as necrotic or apoptotic), and only 9\% of nontreated control cells were in sub- $\mathrm{G}_{0} / \mathrm{G}_{1}$ phase. S-phase cell cycle arrest due to TMZ treatment was observed. Ko143 treatment alone appeared to only slightly change the percentage of apoptotic GBM146 cells or cells with damaged DNA: $10.8 \%$ of cells treated with Ko143 were in sub-G0/G1 phase, whereas $9 \%$ of non-treated control cells were in subG0/G1 phase, as noted. This observation confirmed that Ko143 itself has a minimal effect on the cell cycle of GBM146 cells, making Ko143 a viable option for clinical trials. Analysis showed only a minimal effect of Ko143 $(\sim 1 \%)$ on the cytotoxicity of TMZ (the percentage of cells in sub- $\mathrm{G}_{0} / \mathrm{G}_{1}$ phase increased from $34 \%$ with $\mathrm{TMZ}$ alone to $35 \%$ with $\mathrm{TMZ}+\mathrm{Ko} 143$ ), suggesting that the potentiating effect of Ko143 observed with the ATP-Glo assay was not easily detectable with flow cytometry (Figure 4 ). This could have been the result of different time intervals for each assay or the failure to accurately account for "debris" in flow cytometry after the TMZ+Ko143 treatment, which could have revealed more apoptotic/necrotic cells.

$A B C B 1$ and $A B C G 2$ relative expression levels in Control and TMZ-Resistant phenotypes. The expression of $A B C B 1$ was nearly 43.7-fold greater in GBM146 cells than in GBM9 cells. This may explain why TMZ alone produced a much lower cytotoxic effect in GBM146 cells, as P-gp has been detailed in previous studies to be a major contributor to MDR. Significantly lower expression of the $A B C B 1$ gene within GBM9 cells supported characterization of the GSC line as a much less chemoresistant cell line and thus a negative control. We also found that each cell line displayed very similar levels of $A B C G 2$ expression. In fact, in GBM146 cells, which were declared as TMZ resistant, the expression of $A B C G 2$ was 0.6 -fold lower than that in GBM9
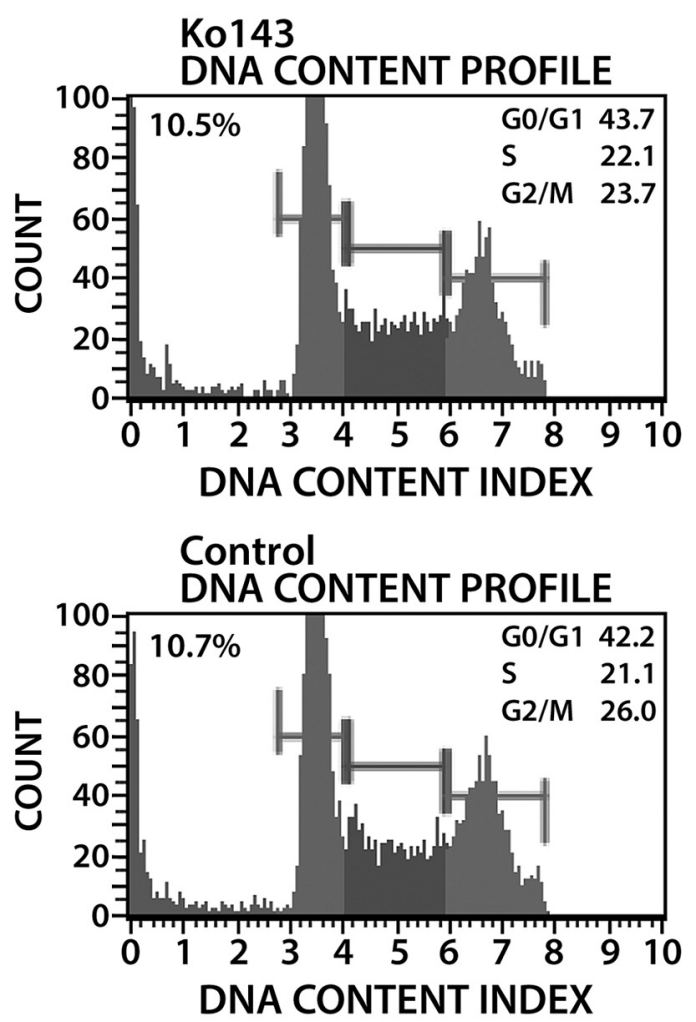

Figure 4. Effects of Kol43 on the cell cycle of temozolomide (TMZ)resistant glioblastoma (GBM) cells. Cell cycle analysis of GBM146 cells treated with Kol43 (5 $\mu \mathrm{M})$ was conducted via flow cytometry alongside a negative control. Cells were fixed with $70 \%$ ethanol, and $48 \mathrm{~h}$ after cell plating their DNA was stained with propidium iodide. Samples were gated to measure only the DNA content of cells exhibiting an appropriate size (debris and cells displaying an abnormal size were discarded). The cell cycle distribution plots were generated by the Milipore Guava Muse. Upon integration of the separate areas below the curve, we observed $10.5 \%$ of cells in the sub- $G_{0} / G_{1}$ phase in the GBM146 cells treated with Ko143 $(5 \mu M)$, which was an insignificant difference when compared to controls (10.7\%).

cells. A likely conclusion is that BCRP is not the only drug efflux pump targeted by Ko143. P-gp may be a primary target of Ko143 within GBM146 cells due to its relatively higher expression.

\section{Discussion}

Previous research has examined the effects of P-gp on antineoplastic drugs and the role it plays in the regulation of their intracellular accumulation (27). BCRP, which was only recently found (in 2012) to be expressed in GBM cells, has also been a topic of recent research (28). Through comparative gene quantification, this study confirmed that P-gp overexpression contributes to MDR in GSCs. We discovered that Ko143 can 
reverse P-gp-mediated chemotherapeutic resistance. ABCG2 expression between GBM9 and GBM146 was similar, yet Ko143 still potentiated TMZ efficacy in the resistant phenotype. Data provided by cell viability analysis indicated that Ko143 does indeed potentiate TMZ efficacy in a chemoresistant phenotype of GSCs. Although previous research outlined the effects of Ko143 on P-gp and BCRP, its effects on those proteins have not been studied with the focus of improving chemosensitivity in GSCs. This research provides a basis for the further exploration of Ko143 and other similar analogs of mycotoxins as a possible way to improve treatment efficacy in chemoresistant GBM patients. Determining TMZ uptake both before and after Ko143 treatment can help confirm the pathways the drug inhibits to reverse MDR. Furthermore, quantification of the absolute (not relative) expressions of $A B C B 1$ and $A B C G 2$ in TMZ-resistant and TMZ-sensitive GBM cohorts will provide further insights into the role they play in MDR.

The MTS assay used in this study contributed to neither data collection nor analysis. Vellonen et al. noted a possible interference between the mechanism of action of the MTT reagent, which is only a slightly less sensitive version of the MTS reagent, and the drug efflux pump inhibitors verapamil (an MDR1 and MRP1 inhibitor) and MK 571 (an MRP1 and MRP2 inhibitor) (26). Therefore, in this study, it was concluded that the results of the MTS assay were inaccurate representations of the effects of Ko143 and TMZ on cell viability. For this study, an ATP-Glo assay was a more fitting choice for assessing cell viability because efflux pumps are inactive in cell lysates.

The use of RT-qPCR confirmed the weak expression of the $A B C G 2$ gene relative to $A B C B 1$ in both GBM9 and GBM146 cells. Though Ko143 has been previously described as a specific BCRP inhibitor with greater than 200-fold selectivity over P-gp, it is likely that Ko143 inhibits P-gp more potently than initially claimed by Allen et al. (25). Moreover, RTqPCR confirmed heightened $A B C B 1$ expression in GBM146 cells relative to GBM9 cells. Flow cytometry results indicate that Ko143 has no cytotoxic effect when administered alone, since only a $1 \%$ increase in apoptotic/necrotic cells was observed compared to a vehicle control. We suggest that the concentration of Ko143 be increased in future experiments $(>5 \mu \mathrm{M})$. Weidner et al. showed that higher concentrations $(20,50,100 \mu \mathrm{M})$ have significantly increased rhodamine 123 (Rh123) penetration in ABCB1 expressing human cells. Whether these concentrations are safe for clinical trials should be determined by future in vivo tests. Given the footing this paper has provided, promising therapies utilizing Ko143 may be developed to address chemoresistance in patients in clinical trials. Discovering a multitude of safe, novel compounds that more generally target drug efflux pumps within MDR-affected GSCs may help play an important role in improving patient prognosis.

\section{Conflicts of Interest}

The Authors declare no conflicts of interest concerning this article.

\section{Authors' Contributions}

Conceptualization and Study Design: SKK, SDL; Execution of Experiments: SKK, SDL, SLL, SK; Data Analysis: SKK, SDL, MSV; Manuscript writing and editing: SKK, SDL, MSV.

\section{Acknowledgements}

Though this study was initially conceptualized by SKK and SDL, the experiments and procedures only materialized through the educational program of the Departments of Neurosurgery and Neuroscience \& Physiology at the State University of New York (SUNY), Upstate Medical University. The authors would like to thank SUNY Upstate Medical University for providing the resources to carry out this study. This work was supported by charitable funding provided by the Debbie's Brain Cancer Research Fund and the George W. Perkins III Research Fund (to MSV).

\section{References}

1 Tamimi AF, Juweid M and De Vleeschouwer S: Epidemiology and outcome of glioblastoma. Glioblastoma Chapter 8, 2017. PMID: 29251870. DOI: 10.15586/codon.glioblastoma.2017.ch8

2 Young RM, Jamshidi A, Davis G and Sherman JH: Current trends in the surgical management and treatment of adult glioblastoma. Ann Transl Med 3(9): 121, 2015. PMID: 26207249. DOI: 10.3978/j.issn.2305-5839.2015.05.10

3 Batash R, Asna N, Schaffer P, Francis N and Schaffer M: Glioblastoma multiforme, diagnosis and treatment; recent literature review. Curr Med Chem 24(27): 3002-3009, 2017. PMID: 28521700. DOI: 10.2174/0929867324666170516123206

4 Stylli SS: Novel treatment strategies for glioblastoma. Cancers (Basel) 12(10): 2883, 2020. PMID: 33049911. DOI: 10.3390/ cancers 12102883

5 Park SH, Kim MJ, Jung HH, Chang WS, Choi HS, Rachmilevitch I, Zadicario E and Chang JW: One-year outcome of multiple blood-brain barrier disruptions with temozolomide for the treatment of glioblastoma. Front Oncol 10: 1663, 2020. PMID: 33014832. DOI: 10.3389/fonc.2020.01663

6 Zhang J, Stevens MF and Bradshaw TD: Temozolomide: mechanisms of action, repair and resistance. Curr Mol Pharmacol 5(1): 102-114, 2012. PMID: 22122467. DOI: $10.2174 / 18744672$ 11205010102

7 Chatterjee N and Walker GC: Mechanisms of DNA damage, repair, and mutagenesis. Environ Mol Mutagen 58(5): 235-263, 2017. PMID: 28485537. DOI: 10.1002/em.22087

8 Marinus MG: DNA mismatch repair. EcoSal Plus 5(1): 10.1128/ecosalplus.7.2.5, 2012. PMID: 26442827. DOI: 10.1128/ecosalplus.7.2.5

9 Wang JYJ: Cell death response to DNA damage. Yale J Biol Med 92(4): 771-779, 2019. PMID: 31866794.

10 Lee SY: Temozolomide resistance in glioblastoma multiforme. Genes Dis 3(3): 198-210, 2016. PMID: 30258889. DOI: $10.1016 /$ j.gendis.2016.04.007 
11 Murayama $\mathrm{T}$ and Gotoh N: Drug resistance mechanisms of cancer stem-like cells and their therapeutic potential as drug targets. Cancer Drug Resist 2: 457-70, 2019. DOI: 10.20517/ cdr.2019.36

12 Chang JC: Cancer stem cells: Role in tumor growth, recurrence, metastasis, and treatment resistance. Medicine (Baltimore) 95(1 Suppl 1): S20-S25, 2016. PMID: 27611935. DOI: 10.1097/ MD.0000000000004766

13 Zhou HM, Zhang JG, Zhang X and Li Q: Targeting cancer stem cells for reversing therapy resistance: mechanism, signaling, and prospective agents. Signal Transduct Target Ther 6(1): 62, 2021. PMID: 33589595. DOI: 10.1038/s41392-020-00430-1

14 Jin X, Jin X and Kim H: Cancer stem cells and differentiation therapy. Tumour Biol 39(10): 1010428317729933, 2017. PMID: 29072131. DOI: 10.1177/1010428317729933

15 Annovazzi L, Caldera V, Mellai M, Riganti C, Battaglia L, Chirio D, Melcarne A and Schiffer D: The DNA damage/repair cascade in glioblastoma cell lines after chemotherapeutic agent treatment. Int J Oncol 46(6): 2299-2308, 2015. PMID: 25892134. DOI: $10.3892 /$ ijo.2015.2963

16 Wijaya J, Fukuda Y and Schuetz JD: Obstacles to brain tumor therapy: Key ABC transporters. Int J Mol Sci 18(12): 2544, 2017. PMID: 29186899. DOI: 10.3390/ijms 18122544

17 Kage K, Tsukahara S, Sugiyama T, Asada S, Ishikawa E, Tsuruo $\mathrm{T}$ and Sugimoto $\mathrm{Y}$ : Dominant-negative inhibition of breast cancer resistance protein as drug efflux pump through the inhibition of S-S dependent homodimerization. Int J Cancer 97(5): 626-630, 2002. PMID: 11807788. DOI: 10.1002/ijc.10100

18 Liu Y, Yang Y, Qi J, Peng H and Zhang JT: Effect of cysteine mutagenesis on the function and disulfide bond formation of human ABCG2. J Pharmacol Exp Ther 326(1): 33-40, 2008. PMID: 18430864. DOI: 10.1124/jpet.108.138115

19 Szöllősi D, Chiba P, Szakacs G and Stockner T: Conversion of chemical to mechanical energy by the nucleotide binding domains of ABCB1. Sci Rep 10(1): 2589, 2020. PMID: 32054924. DOI: 10.1038/s41598-020-59403-7

$20 \mathrm{Yu}$ DM, Huynh T, Truong AM, Haber M and Norris MD: ABC transporters and neuroblastoma. Adv Cancer Res 125: 139-170, 2015. PMID: 25640269. DOI: 10.1016/bs.acr.2014.10.005

21 Cole SP: Multidrug resistance protein 1 (MRP1, ABCC1), a "multitasking" ATP-binding cassette (ABC) transporter. J Biol Chem 289(45): 30880-30888, 2014. PMID: 25281745. DOI: $10.1074 / \mathrm{jbc} . R 114.609248$
22 Tivnan A, Zakaria Z, O’Leary C, Kögel D, Pokorny JL, Sarkaria JN and Prehn JH: Inhibition of multidrug resistance protein 1 (MRP1) improves chemotherapy drug response in primary and recurrent glioblastoma multiforme. Front Neurosci 9: 218, 2015. PMID: 26136652. DOI: 10.3389/fnins.2015.00218

23 Ughachukwu P and Unekwe P: Efflux pump-mediated resistance in chemotherapy. Ann Med Health Sci Res 2(2): 191-198, 2012. PMID: 23439914. DOI: 10.4103/2141-9248.105671

24 Weidner LD, Zoghbi SS, Lu S, Shukla S, Ambudkar SV, Pike VW, Mulder J, Gottesman MM, Innis RB and Hall MD: The inhibitor Ko143 is not specific for ABCG2. J Pharmacol Exp Ther 354(3): 384-393, 2015. PMID: 26148857. DOI: 10.1124/jpet.115.225482

25 Allen JD, van Loevezijn A, Lakhai JM, van der Valk M, van Tellingen O, Reid G, Schellens JH, Koomen GJ and Schinkel $\mathrm{AH}$ : Potent and specific inhibition of the breast cancer resistance protein multidrug transporter in vitro and in mouse intestine by a novel analogue of fumitremorgin C. Mol Cancer Ther 1(6): 417-425, 2002. PMID: 12477054.

26 Vellonen KS, Honkakoski P and Urtti A: Substrates and inhibitors of efflux proteins interfere with the MTT assay in cells and may lead to underestimation of drug toxicity. Eur J Pharm Sci 23(2): 181-188, 2004. PMID: 15451006. DOI: 10.1016/ j.ejps.2004.07.006

27 Callaghan R, Luk F and Bebawy M: Inhibition of the multidrug resistance P-glycoprotein: time for a change of strategy? Drug Metab Dispos 42(4): 623-631, 2014. PMID: 24492893. DOI: 10.1124/dmd.113.056176

28 Bhatia P, Bernier M, Sanghvi M, Moaddel R, Schwarting R, Ramamoorthy A and Wainer IW: Breast cancer resistance protein (BCRP/ABCG2) localises to the nucleus in glioblastoma multiforme cells. Xenobiotica 42(8): 748-755, 2012. PMID: 22401348. DOI: $10.3109 / 00498254.2012 .662726$

Received November 27, 2021

Revised December 23, 2021

Accepted December 27, 2021 